Journal of Patient-Centered

Volume 7

Issue 4 -- Women and Cancer

Article 10

$10-23-2020$

\title{
Conference Proceedings: Aurora Scientific Day 2020
}

Follow this and additional works at: https://aah.org/jpcrr

Part of the Analytical, Diagnostic and Therapeutic Techniques and Equipment Commons, Behavioral Medicine Commons, Cardiology Commons, Diseases Commons, Emergency Medicine Commons, Family Medicine Commons, Geriatrics Commons, Infectious Disease Commons, Integrative Medicine Commons, Internal Medicine Commons, Neurology Commons, Neurosurgery Commons, Oncology Commons, Palliative Care Commons, Primary Care Commons, Public Health Commons, Radiology Commons, Surgery Commons, and the Translational Medical Research Commons

\section{Recommended Citation}

Conference proceedings: Aurora Scientific Day 2020. J Patient Cent Res Rev. 2020;7:355-67. doi: 10.17294/2330-0698.1818

Published quarterly by Midwest-based health system Advocate Aurora Health and indexed in PubMed Central, the Journal of Patient-Centered Research and Reviews (JPCRR) is an open access, peer-reviewed medical journal focused on disseminating scholarly works devoted to improving patient-centered care practices, health outcomes, and the patient experience. 


\section{Conference Proceedings: Aurora Scientific Day 2020}

Abstracts published in this supplement were among those presented at the 46th annual Aurora Scientific Day research symposium, held virtually on May 20, 2020. The symposium provides a forum for describing research studies conducted by faculty, fellows, residents, and allied health professionals affiliated with Wisconsin-based Aurora Health Care, a part of the Advocate Aurora Health health system, which publishes the Journal of Patient-Centered Research and Reviews.

\section{ORAL PRESENTATIONS}

Machine Learning Models for Distinguishing Hypertrophic Cardiomyopathy From Amyloidosis Using Body Surface Electrocardiograms

Christopher R. Beal, M. Fuad Jan, Arshad Jahangir, A. Jamil Tajik

Advocate Aurora Research Institute; Aurora Cardiovascular and Thoracic Services, Aurora St. Luke's Medical Center

Background: The echocardiogram is the current gold standard for diagnosing hypertrophic cardiomyopathy (HCM) and amyloidosis. The equipment and medical expertise required to perform this procedure and interpret results can be costly and relatively scarce in some health care systems. The 12-lead electrocardiogram (ECG) is a widely available, lower-cost tool to establish baseline cardiac function. If demonstrated to be an accurate method for diagnosing $\mathrm{HCM}$ and amyloidosis, there is potential to expand the diagnostic capabilities of the ECG for these diseases.

Purpose: To demonstrate the viability of using machine learning models to diagnose patients with HCM and/or amyloidosis from 12lead ECG data by assigning them to one of two disease groups.

Methods: We analyzed ECG parameters that were extracted from raw ECG data by the MUSE database. We selected 19 patients who had undergone previous echocardiograms: 10 patients with HCM contributed 116 ECG scans, and 9 patients with amyloidosis contributed 181 ECG scans. A total of 78 ECG parameters were analyzed from each 12-lead ECG trace, resulting in nearly 300,000 data elements analyzed in this study. The dataset was split into two parts: $75 \%$ of the data was used to train the models, and the remaining $25 \%$ was used to validate results. Several different machine learning models, including neural networks, support vector machines, and random forests, were evaluated. Model performance was measured in terms of accuracy, sensitivity, and specificity.

Results: The results from our analysis demonstrate that machine learning models can distinguish HCM from amyloidosis using 12lead ECG data. Random forests appeared to be the most appropriate model (accuracy up to $99.80 \%$ ), while the neural network model was the least accurate (96.43\%). All models had sensitivity and specificity values greater than 0.96 .

Conclusion: Hypertrophic cardiomyopathy can be distinguished from amyloidosis by machine learning models using 12-lead electrocardiographic data. Machine learning has the potential to automate and reduce the cost of diagnosing these cardiac diseases in more health care systems. Future work will use this approach to determine if HCM can be distinguished from structurally normal hearts and/or other cardiac diseases.
Evaluating Soluble AXL as a Potential Serum Biomarker for Glioblastoma

Daniel Raymond, Melanie B. Fukui, Robert J. Winn, Amber LaCrosse, Natalie Polinske, Brittany Last, Beau Adams, Sam D. Zwernik, Richard A. Rovin, Parvez Akhtar

Advocate Aurora Research Institute; Aurora Neuroscience Innovation Institute, Aurora St. Luke's Medical Center; Upper Michigan Brain Tumor Center, Northern Michigan University; Biorepository and Specimen Resource Center, Aurora St. Luke's Medical Center

Background: We previously found that the cell surface receptor AXL is overexpressed in glioblastoma (GBM). AXL expression contributes to both a malignant and metastatic phenotype. While AXL is abundant in GBM, its expression in normal tissue is low or undetectable. The extracellular portion of AXL is enzymatically cleaved, releasing soluble AXL (sAXL). sAXL is detectable in serum and tumor-related fluid collections and has been described in melanoma and hepatocellular carcinoma. The relationship between sAXL and GBM is unclear.

Purpose: To determine if sAXL can be detected in serum from GBM patients and if there is a correlation with extent of disease.

Methods: We used 84 serum samples from 25 patients with GBM and 42 samples from 42 healthy volunteers, which were diluted 1:200 in low-cross buffer and phosphate-buffered saline. Aliquots were stored at $-80^{\circ} \mathrm{C}$. Samples in low-cross buffer were tested using a commercially available AXL ELISA kit, and the samples in phosphate-buffered saline were tested for total protein using a commercially available bicinchoninic acid assay. Tumor tissue from 15 of the enrolled patients was evaluated for AXL expression using western blotting. Interpretation of tumor volume was performed using T1 magnetic resonance imaging scans by a trained neuroradiologist. Student's $t$-test and linear regression were performed to determine significance of variation.

Results: The mean serum sAXL concentration was $35.77 \pm 1.25$ $\mathrm{ng} / \mathrm{mL}$ in the GBM samples compared to $29.99 \pm 1.80 \mathrm{ng} / \mathrm{mL}$ in the healthy controls $(t(\mathrm{df})=2.652(124) ; \mathrm{P}=0.009)$. Controlling for total protein further distinguished the two groups significantly. The difference between pre- and postoperative sAXL concentrations was not statistically significant. There was a slightly negative correlation between SAXL concentrations and corresponding preoperative tumor volumes. Comparing sAXL concentrations to the AXL expression in the tumor lysates yielded a stronger negative correlation.

Conclusion: While serum sAXL concentration is significantly higher in patients with glioblastoma compared to controls, in this small sample, there was not a significant correlation between SAXL 
levels and volume of GBM. Evaluating sheddase enzyme expression in the GBM lysates is needed to confirm the lack of correlation between sAXL in the serum to AXL found in GBM tumor tissue.

\section{Buprenorphine Therapy for Opioid Use Disorder in a Rural Family Medicine Residency Clinic: Outcomes}

Shivani Bhatnagar, Eftu Boru, Munish Kaushik, Jace Varkey, Marianne Klumph, Jeffrey Tiemstra

Family Medicine, Aurora Lakeland Medical Center; Aurora UW Medical Group; Center for Urban Population Health

Background: Opioid use disorder (OUD) is a national epidemic. Office-based medication-assisted treatment (MAT) with buprenorphine, a partial opioid agonist, is effective and can be offered by primary care physicians to increase patient access.

Purpose: To assess effectiveness of MAT for OUD in a rural family medicine clinic and identify factors associated with patient discontinuation/drop-out of treatment.

Methods: The study was a retrospective chart review of all 123 MAT patients entering treatment for OUD at Aurora Lakeland Family Medicine Clinic from January 1, 2018, to July 1, 2019. Data was collected for the first 6 months or until discontinuation. Variables studied included basic demographics, opioid first used, history of heroin use, continued use of illicit/nonprescribed drugs, maintenance MAT dose, and involvement in behavioral health treatment (BHT). Data was analyzed using regression analysis and Fisher's 2-tailed exact test.

Results: Mean age was 38.7 years; 52\% were female, $48 \%$ male. Mean time in MAT was 21 weeks. In all, $63 \%$ of patients were in MAT at 6 months; $58 \%$ had remained opioid-free; $65 \%$ had started with prescription opioids; $63 \%$ had ever used heroin; and $43 \%$ had ever used intravenous heroin. Factors associated with MAT discontinuation included cocaine in urine drug screen (UDS) $(\mathrm{P}=0.049)$, nonprescribed opioids in UDS $(\mathrm{P}<0.001)$, and lack of BHT (dropout rate: $15 \%$ for BHT vs $52 \%$ for no BHT; $\mathrm{P}<0.001$ ). Factors not associated with MAT discontinuation included age $(\mathrm{P}=0.407)$, gender $(\mathrm{P}=0.454)$, history of heroin use $(\mathrm{P}=0.244)$ or intravenous heroin use $(\mathrm{P}=0.575)$, alcohol in UDS $(\mathrm{P}=0.515)$, nonprescribed stimulants in UDS $(\mathrm{P}=0.445)$, nonprescribed benzodiazepines in UDS $(\mathrm{P}=0.201)$, and maintenance buprenorphine dose $(\mathrm{P}=0.890)$.

Conclusion: Over half of patients remained in medicationassisted treatment and opioid-free. Cocaine and illicit opioid use were associated with decreased retention in MAT, while alcohol, nonprescribed stimulant, and nonprescribed benzodiazepine use were not. Participation in behavioral health treatment was associated with significantly higher retention in MAT, although $48 \%$ of patients not receiving behavioral health treatment also remained in MAT.

National Trends in Delirium Rates of Hospitalized Older Adults With Heart Failure in the United States (1998-2014)

Ariba Khan, Maharaj Singh, Nasir Sulemanjee, Arshad Jahangir, Marianne Klumph, Alexander Schwank, Michael L. Malone

Geriatrics, Aurora Sinai Medical Center; Aurora UW Medical Group; Advocate Aurora Research Institute; Marquette University School of Dentistry; Aurora Cardiovascular and Thoracic Services, Aurora St. Luke's Medical Center; Center for Urban Population Health
Background: Delirium is common $(17 \%-38 \%)$ in hospitalized older adults with heart failure (HF). Improved care in the field of cardiology, along with a decline in HF hospitalizations, may result in a reduction of delirium associated with HF. However, delirium is often underrecognized, so the recent national efforts to improve delirium recognition may result in an increase in delirium diagnosis. Purpose: To compare the trends in delirium rates over a period of 16 years in patients admitted with a primary diagnosis of $\mathrm{HF}$ and to determine their associated factors.

Methods: The Healthcare Cost and Utilization Project's National Inpatient Sample (NIS) database was used to identify older adults ( $\geq 65$ years of age) with a diagnosis of $\mathrm{HF}$ and delirium from 1998 to 2014. The NIS is a representative sample of $20 \%$ of national hospitalizations. ICD-9 diagnostic codes were used to identify delirium and primary diagnosis of HF. The trend in the rates of HF were then compared to patients with and without delirium. Regression models and chi-squared tests between groups were used to determine statistical differences.

Results: Out of 1,820,818 patients with a diagnosis of HF, $61 \%$ were White, $56 \%$ female, and 4\% hospital mortality. Mean age was 79 years. Delirium diagnosis was identified in 28,937 (1.6\%) patients. There was a reduction in the proportion of HF admissions that suggested a decline in HF over the study time period (1998 vs 2014; $\mathrm{P}<0.001)$. In addition, although not as drastic, there was a drop in HF admission with a delirium diagnosis (1998 vs 2014; $\mathrm{P}<0.001)$. Patients with delirium, when compared to those without delirium, had a longer length of stay ( 7 days vs 5 days; $\mathrm{P}<0.001$ ), had higher mortality ( $13 \%$ vs $5 \% ; \mathrm{P}<0.001)$, and were more likely to be discharged to a nursing home ( $34 \%$ vs $20 \%$; $<<0.001)$. Delirium diagnosis was more common in Whites $(64 \%$ vs $36 \%$; $\mathrm{P}<0.001)$ and less likely in females ( $47 \%$ vs $53 \%$; $\mathrm{P}<0.001)$. Variables associated with delirium in logistic regression analysis included male sex, white race, age, length of stay, dementia diagnosis, admission to a teaching hospital, elective admission, and Charlson/Deyo comorbidity score (area under the curve: 0.64).

Conclusion: Overall, primary heart failure alone illustrated a substantial decrease over the 16-year time period. However, the rate of delirium in older patients with primary HF was not only much lower than expected but remained stable in the same time period. Most importantly, those with delirium had higher inpatient mortality and length of stay as compared to those who did not have delirium.

\section{POSTER PRESENTATIONS}

Incidence of Vitamin D Deficiency in the Internal Medicine Clinic at Aurora Sinai Medical Center

Daniel Mundt, Wajih Askar, Kayla Heslin, Marianne Klumph, Kern A. Reid

Internal Medicine, Aurora Sinai Medical Center; Advocate Aurora Research Institute; Aurora UW Medical Group; Center for Urban Population

Background: According to the Centers for Disease Control and Prevention, $10 \%$ of the U.S. population has severe vitamin D deficiency (VDD), with African Americans having the highest prevalence of low vitamin D concentration. Studies have shown that VDD is significantly higher among urban midwestern populations. The detrimental effects of VDD on the bone are well known; however, recent studies suggest that VDD also might be involved in the immune, cardiovascular, and neurological system. Nevertheless, 
there is still debate surrounding who, how, and how often individuals should be screened for VDD.

Purpose: To assess the prevalence of VDD in one internal medicine clinic and identify risk factors.

Methods: Data were retrospectively collected on unique adult patients $(\geq 18$ years old) who attended the clinic at any point from January 2018 to December 2018. Vitamin D levels of $\geq 30 \mathrm{ng} / \mathrm{ml}$ were considered normal, while levels of $<30 \mathrm{ng} / \mathrm{ml}$ were considered deficient. Patients who had more than one vitamin D test were tracked for further analyses. Basic descriptive statistics were used to describe the population. Chi-squared tests and $t$-tests were used as appropriate to compare groups.

Results: Of the patient cohort ( $\mathrm{n}=3,976)$, only $17.56 \%$ had vitamin D levels tested and $12 \%$ had a prior diagnosis of VDD. Of those tested, $68 \%$ were females, $72 \%$ were African Americans, and the average age was 59 years. Unlike race $(\mathrm{P}=0.80)$, women, patients with a previous diagnosis of bone fracture, and those with a current diagnosis of alcohol use disorder, celiac disease, or chronic kidney disease were significantly more likely to have vitamin D levels tested $(\mathrm{P}<0.01)$. As expected, individuals were more likely to be tested in winter than in any other season (42\%), and the majority of patients tested had VDD (71\%), including individuals with chronic kidney disease $(\mathrm{P}=0.002)$. However, there were no differences in VVD based on race $(\mathrm{P}=0.464)$. In addition, if a patient was re-tested, they were more likely to show improvement of vitamin D levels (42\%), although $28 \%$ stayed the same.

Conclusion: Although African Americans are generally known to have lower levels of vitamin D when compared to other races, we found they were not more likely to be tested nor have more vitamin $\mathrm{D}$ deficiency. Improvement of vitamin D levels for those re-tested indicates that interventions are being utilized. Parallel to results previously reported, patients with chronic kidney disease are more likely to have VDD and could benefit from annual testing for vitamin D levels. Awareness of these differences could help to lower rates of VDD in this at-risk population.

\section{Impact of Goals of Care Conversation Training}

Temu Brown, Marianne Klumph, Kayla Heslin, Elizabeth A. Duthie, Tim Jessick

Hospice and Palliative Medicine, Aurora St. Luke's Medical Center; Aurora UW Medical Group; Center for Urban Population Health; Advocate Aurora Research Institute; University of Wisconsin-Milwaukee; Palliative Care, Aurora West Allis Medical Center

Background: Palliative interventions by nonpalliative health care providers such as hospitalists have proved to be beneficial to patients. A primary task of these providers is to hold goals of care (GOC) conversations with patients early during their hospital stay. This can help reserve the expertise of palliative care specialists for more complex patients. While a previous local evaluation focused on providers' overall comfort in initiating GOC conversation before and after an intervention, no study has explored whether the intervention resulted in providers having more GOC conversations.

Purpose: To explore whether GOC training sessions increased hospitalists' utilization of the GOC dotphrase generated by the Epic electronic health record; also, to evaluate if there was an improvement in the overall quality of GOC conversations held with patients upon admission.

Methods: Aurora Health Care hospitalists participated in training sessions covering GOC conversations during 2017-2018. We reviewed 200 history and physical notes (H\&Ps) for 5 full-time hospitalists before and after training. H\&Ps were reviewed only for patients who were $\geq 65$ years of age and admitted directly to the intensive care unit. For each hospitalist, only the 20 most recent charts before and after the intervention were included. Documented GOC conversations were tabulated, including the use of the GOC dotphrase. Quality assessment was done by searching for goalconcordant keywords and their synonyms within these conversations. Basic descriptive statistics were used to describe the population. Chisquared tests and $t$-tests were used as appropriate to compare groups. Results: Patient demographics of H\&Ps reviewed were as follows: 95\% White, 59\% female, and mean age 79 years. Older patients (mean: 84 years) were more likely to have a GOC conversation $(\mathrm{P}<0.001)$. Although there was an increase in usage of the GOC dotphrase after training sessions ( $73 \%$ compared to $31 \%$; $\mathrm{P}=<0.01$ ), only $23 \%$ of patients had a GOC conversation. The overall quality of the GOC conversations both before and after the intervention remained essentially unchanged (17\% vs $18 \%)$.

Conclusion: Half-day training sessions in conducting goals of care conversations were associated with an increased usage of the GOC dotphrase. Our next steps will be to broaden the scope of this study by not only retraining hospitalists but also including providers from other facilities in our analysis, thus increasing the number of analyzed patient history and physical notes.

\section{Offering Acupuncture to Patients in the Emergency Department Continues to Decrease Acute Pain}

John R. Burns, Jessica J. F. Kram, Jeanne M. Stark Casadont, Vashir Xiong, Nancy Conway, Dennis J. Baumgardner

Integrative Medicine, Aurora Sinai Medical Center; Aurora UW Medical Group; Center for Urban Population Health; Department of Family Medicine, Aurora UW Medical Group

Background: Acupuncture has been associated with improving various types of acute pain. Our previous study found that acupuncture administered in the emergency department (ED) setting decreases acute pain regardless of analgesics received during visit.

Purpose: To determine if 2 years after program onset, acupuncture continues to improve pain scores regardless of analgesics used during ED visits.

Methods: We retrospectively reviewed patients from 2019 who accessed our ED acupuncture program. Similar to our previously published work in 2017, acupuncture services were offered to adult patients ( $\geq 18$ years old) based on their emergency severity index (ESI; highest severity [1] - lowest severity [5]), reason for visit, and physician recommendation. Only patients' first visit in 2019 was included in analyses. Basic summary statistics were used to describe patient characteristics. Paired $t$-tests were used to determine differences in pre- and postacupuncture pain, stress, anxiety, and nausea scores (ie, no pain [0] - worst pain [10]). Logistic regression models were used to determine associations between improvements in pain and patient or visit characteristics.

Results: Acupuncture services were provided to 199 patients (mean age: 47.5 years; body mass index: $32.0 \mathrm{~kg} / \mathrm{m}^{2}$ ), who were predominately female $(78.4 \%)$ and non-Hispanic White $(70.4 \%)$ and who had an ESI score of $3(63.3 \%)$ or $4(31.2 \%)$. As in 2017, mean pre- and postacupuncture scores for pain (6.7 vs 3.4), stress (6.3 vs 1.5 ), anxiety ( 4.9 vs 1.2 ), and nausea (1.5 vs 0.4 ) significantly decreased $(\mathrm{P}<0.001$ for all). Most patients had a final acute pain- 
related diagnosis for neck/back pain (41.9\%), abdominal pain (18.7\%), or head/headache (14.7\%). Improved pain scores were not associated with any patient characteristics. As in 2017, receiving opioids only during the ED visit was not associated with improved pain scores $(\mathrm{P}=0.21)$, nor was receiving nonsteroidals $(\mathrm{P}=0.07)$ or tramadol $(\mathrm{P}=0.42)$. However, receiving any pain medication in the ED was associated with improved pain scores $(\mathrm{P}=0.028)$. Improved pain scores was not predictive for receiving any pain medications, including opioids, at discharge ( $\mathrm{P}>0.05$ for all).

Conclusion: Similar to 2017 findings, emergency department acupuncture remains associated with significantly decreased pain, stress, anxiety, and nausea. Our findings continue to support ED acupuncture for acute pain in the ED and further support the need for a larger randomized controlled trial.

\section{Assessment of Decentralized, Inpatient Pharmacist Blood Culture Audit and Provider Feedback}

\section{Brittany Brown, Thomas J. Dilworth}

\section{Aurora Pharmacy, Advocate Aurora Health}

Background: Hospitalized patients with blood stream infections (BSI) are at increased morbidity and mortality risk despite advances in therapy and overall care. Audit and provider feedback for positive blood cultures by pharmacists has been shown to improve care and outcomes for patients with BSI. However, previous studies employed pharmacy specialists with infectious diseases (ID) training in a centralized audit and feedback model. It is unknown if decentralized inpatient pharmacists, with support from ID pharmacists as needed, could impact patient care similarly.

Purpose: To evaluate antibiotic treatment and outcomes for patients with BSI for whom blood culture audit and provider feedback was performed by decentralized inpatient pharmacists.

Methods: This was a retrospective review of 100 adult patients with BSI in Aurora Health Care with a positive blood culture from April 1, 2019, to June 30, 2019. Data collected included patient demographics, blood culture draw time, culture results (gram stain, pathogen, antibiotic susceptibilities), time to empiric antimicrobial therapy start, and time to antibiotic optimization. The primary outcome assessed was time from blood culture draw to effective empiric antimicrobial therapy. The secondary outcome assessed was time from blood culture draw to optimal antimicrobial therapy. Outcomes collected were compared to previously published studies. Results: 100 patients with positive blood cultures were included. Urine was the predominant BSI source (47\%), followed by skin/ soft tissue (14\%). The most common pathogens were gramnegative bacilli (61\%). A total of 8 multidrug resistant organisms were identified. The median time to effective antibiotic therapy from blood culture draw was 2 hours (interquartile range [IQR]: 1-4.75). The median time to optimal definitive antibiotic therapy was 36 hours (IQR: 2.13-64). These results are similar to previously published studies of ID pharmacy specialists in a centralized audit and feedback model.

Conclusion: Audit of blood stream infections and provider feedback by decentralized inpatient pharmacists, along with as-needed support from infectious disease pharmacists, may represent an alternative to the centralized ID pharmacist model. Application of these findings to practice will allow for more efficient use of pharmacist time and expand decentralized inpatient pharmacy practice.
Fruit and Vegetable Vouchers Do Not Increase Redemption or Consumption

Andrew McAsey, Priti Rana, Yelena Goschin, Lisa Sullivan Vedder, Jessica J. F. Kram, Marianne Klumph

University of Wisconsin School of Medicine and Public Health; Family Medicine, Aurora St. Luke's Medical Center; Family Medicine, Aurora Sinai Medical Center; Aurora UW Medical Group; Center for Urban Population Health

Background: It is well known that a diet rich in fresh fruits and vegetables improves health outcomes. In central Milwaukee, specifically the 53233 zip code area, there is a limited supply of vendors that sell fresh produce to its residents.

Purpose: To increase produce consumption among patients through a fruit and vegetable prescription (FnVRx) program.

Methods: In 2019, patients at 1 family medicine residency clinic in the 53233 zip code were recruited and assigned to one of two groups. The study group was given a packet of materials including $2 \$ 20$ prescription vouchers for fresh produce redeemable at the nearby (1.7 miles from clinic) Fondy Farmers Market, along with a verbal explanation of packet contents, while the control group was given the same packet but without verbal overview. Groups were assigned based on availability of study team member to discuss packet contents. Participants completed a baseline assessment at their initial visit; follow-up phone surveys were completed at 4 and 8 weeks post-office visit. Basic descriptive statistics were performed, and Fisher's exact tests were used for $2 \times 2$ tables.

Results: Of the total participants ( $\mathrm{N}=107), 71 \%$ met food insecurity criteria, $46 \%$ had an income of $<\$ 15,000$, and $89 \%$ shared food with $\geq 2$ family members. Participants were predominantly female (86\%), African American (72\%), and in the control group (77\%). Overall, 71\% never redeemed a FnVRx. Of those surveyed at 4 weeks postrecruitment, $31 \%$ of individuals and $19 \%$ of households recalled consuming more produce, as opposed to $19 \%$ of individuals and $16 \%$ of households who had recalled more produce consumption at 8 weeks. No statistical difference was associated with redemption of FnVRx in regard to gender, income level, prior use of farmers' markets, groups, presence of food insecurity, or size of household ( $\mathrm{P}>0.05$ for all). However, increasing age was associated with FnVRx redemption $(\mathrm{P}=0.016)$. Overall, the most commonly cited reasons for not redeeming FnVRx were "too busy" or "forgot."

Conclusion: The solution to increasing fresh produce consumption is multifactorial. The answer is not as simple as providing individuals with monetary-based prescriptions for farmers' markets. Many barriers likely prevent increased produce consumption (eg, time, motivation, transportation, cultural preferences, distance to farmers' markets, and education). Despite low redemption rates, this project identified a need for food resources for our patients and further development of interventions to meet those needs.

\section{Zika Virus Targets Stem Cell Markers and Modulates Antiviral Responses in Glioblastoma Cancer Stem Cells}

Beau Adams, Sam D. Zwernik, Daniel Raymond, Richard A. Rovin, Parvez Akhtar

Advocate Aurora Research Institute; Aurora Neuroscience Innovation Institute, Aurora St. Luke's Medical Center 
Background: Glioblastoma is the most common and lethal form of brain cancer. Standard treatment involves surgery, chemotherapy, and radiation. Tumor recurrence is caused by a population of glioblastoma cancer stem cells (GSCs) that resist and survive treatment. There are currently no pharmacological agents available for specific targeting of GSCs. Zika virus (ZIKV) is a flavivirus that targets normal neural stem cells in the developing brain and causes microcephaly. ZIKV also selectively targets GSCs in a similar manner. We examined the effect of ZIKV on specific stem cell markers as well as antiviral responses in patient-derived GSCs. Purpose: To understand the oncolytic mechanism of ZIKV toward GSCs.

Methods: Glioblastoma patient-derived cell lines were acquired from within our institution and used for the entirety of this study. Patient-derived cell lines were grown in spheres using supplemented neurocult media. ZIKV strain MR766 was propagated in Vero cells, and viral stock was titrated by plaque assays. Glioblastoma patient cell lines were infected with ZIKV at a multiplicity of infection of 1. The percentage of infection was quantified by flow cytometry using a pan-flavivirus antibody. Western blotting was used to characterize protein expression, while qRT-PCR was used to quantify gene expression pre- and post-ZIKV infection in patient-derived cell lines. Results: We tested multiple stem cell markers and found that Sox2 and Nestin expression is highly upregulated in our glioblastoma patient-derived cell lines $(7714,7730,7753)$, while Nanog, Oct-4, and Musashi-1 were at basal levels. We further found that both Sox2 and Nestin were significantly decreased post-ZIKV infection. Next, we found that ZIKV induces antiviral responses postinfection through an increase of interferon-induced genes IRF1, IFITI, ISG15, and IL6. Lastly, we found increased expression of FGF2 and Caspase-3 post-ZIKV infection, which are involved in cell differentiation and cell death, respectively.

Conclusion: Our results suggest that Zika virus infection causes loss of glioblastoma stem cell self-renewal through decreased Sox2 and Nestin expression. Furthermore, ZIKV leads to antiviral responses in GSCs through an increase of multiple interferon-induced genes. Finally, genes involved in cell differentiation and cell death are upregulated in GSCs post-ZIKV infection. Thus, there are multiple mechanisms to explain GSC death following ZIKV infection.

\section{p53 Level and MGMT Promoter Methylation Are Playing a Role in Zika Virus Replication in Glioblastoma}

Sam D. Zwernik, Beau Adams, Jun Yin, Santhi D. Konduri, Richard A. Rovin, Parvez Akhtar

\section{Advocate Aurora Research Institute; Aurora Neuroscience Innovation Institute, Aurora St. Luke's Medical Center}

Background: Glioblastoma (GBM) is a malignant primary brain cancer. The poor median survival rate for patients with GBM of 15 months has not budged for the past 15 years, when the current standard treatment was first approved. There is no standard-of-care chemotherapy for recurrent GBM. Needless to say, novel treatments and treatment strategies for GBM are needed. One such novel treatment strategy is an oncolytic virus. The Zika virus' (ZIKV) affinity for fetal neural stem cells has made it a compelling candidate as an oncolytic therapy for GBM. Previous studies have shown that ZIKV does infect and replicate in most but not all GBM cancer cells. Understanding the genetic milieu that is permissive for ZIKV infection is critical to the creation of a safe and effective viral oncolytic treatment. This report presents initial data for a ZIKV replication gene signature.
Purpose: We hypothesized that p53 level and MGMT promoter methylation are playing a role in ZIKV replication in GBM cancer cells.

Methods: ZIKV strain MR766 was propagated in Vero cells. Viral stock was titrated by plaque assays. Western blot was used to characterize MGMT, AXL, p53, and Sox2 expression in 8 commercial GBM cell lines (LN229, U87, A172, U251, LN18, T98G, U137, and U118). The cell lines were stratified by MGMT promoter methylation status and were exposed to ZIKV at a multiplicity of infection of 1 . The percentage of infected cells was quantified by flow cytometry using the pan-flavivirus anti-E protein Ab 4G2. We also compared RNA-seq data of ZIKV replicating and nonreplicating GBM cell lines to find differentially expressed genes. Results: In this study, we found that AXL was overexpressed in all 8 commercial GBM cell lines. With flow cytometry, we found that productive ZIKV replication only occurs in the MGMT-methylated (LN229, A172, U87, and U251) cell lines and not in MGMTunmethylated (T98G, LN18, U118, and U138) cell lines. qRT-PCR data showed that ZIKV enters all 8 cell lines but can only replicate in MGMT-methylated cell lines. Additionally, p53 expression trended lower in MGMT-methylated cell lines. RNA sequencing data comparing ZIKV replicating and nonreplicating GBM lines has identified multiple differentially genes.

Conclusion: Based on these results, there is a clear difference in the ability of the Zika virus to replicate in glioblastoma cell lines based on MGMT and p53 expression. Additional work is underway to understand the mechanism(s) underlying these findings and to define a ZIKV replication gene signature.

\section{Data in the Electronic Health Record Can Be Used at the Bedside to Identify Older Hospitalized Patients With Delirium}

Ariba Khan, Kayla Heslin, Michelle Simpson, Michael L. Malone

Geriatrics, Aurora Sinai Medical Center; Aurora UW Medical Group; Advocate Aurora Research Institute; Center for Urban Population Health; Ed Howe Center for Research, Aurora Sinai Medical Center

Background: Delirium is common among hospitalized older adults and associated with adverse outcomes. Delirium remains underrecognized, and efforts are focused on early recognition and prediction. While several delirium predictive rules have been developed, only a handful have focused on electronic health record (EHR) data. The coupling of prediction rules with features of the EHR are in their infancy but hold promise in their ability to aid in identification of delirium.

Purpose: To determine variables within our health system's EHR that can be used to identify older hospitalized patients with delirium. Methods: This is a prospective study among hospitalized patients ( $\geq 65$ years of age) from February 2016 to November 2017. Patients were excluded if they: 1) were non-English-speaking, comatose, ventilated, or combative; 2) were intensive care or surgical patients; or 3) had severe aphasia, severe dementia, or a critical illness. Researchers screened daily for delirium using the 3-minute diagnostic confusion assessment method (3D-CAM). Predictive variables were extracted from the EHR. Basic descriptive statistics were conducted. Chi-squared and Fisher's exact tests were used to compare differences among those diagnosed with or without delirium. Binary logistic regression was used for multivariable modeling. 
Results: Among 408 participants, mean age at admission was 75 years, $61 \%$ were female, and $83 \%$ were African American. The overall rate of delirium was $16.7 \%$ (prevalent delirium: $10.5 \%$ $[n=43]$; incident delirium: $6.1 \%[n=25])$. There was no statistical difference in 30 -day mortality ( $2.9 \%$ vs $2.7 \%$ ) or 30 -day readmission rates $(13.2 \%$ vs $14.7 \%)$ between those with and without delirium ( $\mathrm{P}>0.05$ for both). Even so, patients with delirium were older, more likely to have a diagnosis of infection and/or cognitive impairment, and more likely to have increased severity of illness ( $\mathrm{P}<0.05$ for all). Moreover, patients with delirium had a lower Braden (pressure ulcer risk) score and higher Morse fall score ( $\mathrm{P}<0.01$ for both). In multivariable analysis, cognitive impairment (odds ratio: 5.49; $95 \%$ CI: $2.77-10.87$ ) and lower Braden scores (odds ratio: 1.29; 95\% CI: 1.18-1.41) remained significant predictors of delirium among hospitalized patients.

Conclusion: Our study found that cognitive impairment and lower Braden scores were associated with hospital delirium. Further research is needed to develop an automated, dynamic (daily) prediction model inclusive of these variables.

\section{Usefulness of Procalcitonin in Aiding Physician Assessment and Treatment of Potential Serious Bacterial Infections}

Jennifer K. Homa, Taylor A. Romdenne, Alex D. Christl, Ashley R. Heesacker, Darren M. Heesacker

Advocate Aurora Research Institute; Emergency Department, Aurora BayCare Medical Center; Emergency Department, BayCare Clinic; Emergency Medicine, BayCare Clinic

Background: Procalcitonin (PCT) is used as a biomarker for the diagnosis of serious bacterial infections (SBI). To date, studies have not compared PCT to clinical judgment and it remains unclear whether PCT adds to the physician's clinical judgment when diagnosing SBI.

Purpose: To evaluate the diagnostic usefulness of PCT in comparison to blood culture results and the physician's clinical judgment in patients presenting to the emergency department with signs of SBI.

Methods: A prospective cohort study was conducted with 400 patients suspected of having an SBI who presented to the emergency department at 2 community hospitals in Wisconsin from 2016 to 2018. PCT was performed on all patients in addition to the standard of care (SOC) for suspected SBI. PCT results were not available to the physicians throughout the duration of the study. Physicians completed a brief survey that asked if they thought the patient was septic upon ordering SOC labs and again after they reviewed the SOC lab results. Data were collected to determine if patients were diagnosed with an SBI during their stay. Multivariate logistic regression was used to examine factors associated with an SBI diagnosis.

Results: Among the patients, 186 (46.5\%) were diagnosed with an SBI during their hospital stay. High serum levels of PCT ( $\geq 0.25 \mathrm{ng} /$ $\mathrm{mL}$ ) were an independently significant predictor for an SBI diagnosis in patients with signs of infection (odds ratio [OR]: $1.96,95 \% \mathrm{CI}$ : 1.13-3.39; $\mathrm{P}=0.016$ ). In addition, patients suspected of having an SBI are 2.62 times more likely to be diagnosed with an SBI when the blood culture result is positive (OR: 2.62, 95\% CI: 1.19-5.77; $\mathrm{P}=0.017$ ) and 7.13 times more likely to be diagnosed with an SBI when the physician believes the patient is septic after reviewing the SOC lab results (OR: 7.13, 95\% CI: 3.64-13.97; $\mathrm{P}<0.001$ ). There was no association between the physician's clinical judgment before reviewing the SOC lab results and SBI diagnosis (OR: 1.74, 95\% CI: 0.88-3.45; $\mathrm{P}=0.111$ ). None of the other factors, including lactic acid, were found to be significant predictors for an SBI diagnosis.

Conclusion: Procalcitonin, blood culture results, and clinician judgment after reviewing standard-of-care labs provide important diagnostic value when diagnosing serious bacterial infections. Clinician judgment before reviewing SOC lab results was not associated with an SBI diagnosis; thus, SOC labs do have added value in aiding physician assessment of potential SBI. This study offers a unique perspective as, to date, no other studies have compared PCT results to clinical judgment.

\section{Goals of Care Conversations: A Training Program Evaluation}

Elizabeth A. Duthie, Colleen M. Galambos, Theresa Frederick, Jacob Verkuilen, Tim Jessick

University of Wisconsin-Milwaukee; Center for Urban Population Health; Helen Bader School of Social Welfare, University of Wisconsin-Milwaukee; Continuing Professional Development, Advocate Aurora Health; Palliative Care Medicine, Aurora West Allis Medical Center

Background: Patients benefit when their health care providers engage in conversations about treatment options and goals of care. However, these conversations frequently do not occur. Researchers have called for new approaches to increase such conversations. The Teaching Primary Palliative Care Training program is one such approach.

Purpose: To determine whether Teaching Primary Palliative Care Training increases participant comfort engaging in goals of care conversations with seriously ill and dying patients shortly after the training and 1 year after the training.

Methods: 78 health care providers participated in Teaching Primary Palliative Care Training during the fall of 2018. Participants completed a self-assessment in which they reported on their comfort level in various circumstances related to the management of seriously ill and dying patients at baseline before the training, shortly after completing the training, and again 1 year later. We used the 1-sided sign test to assess for statistically significant improvement in selfreported comfort.

Results: At the first follow-up, self-assessments reflected significant improvement in comfort, compared to baseline, in all 5 circumstances related to the management of seriously ill and dying patients. These circumstances included: delivering bad news $(\mathrm{P}=004)$; discussing $\mathrm{CPR} / \mathrm{DNR}(\mathrm{P}=003)$; discussing hospice or palliative care referral $(\mathrm{P}=001)$; discussing artificial hydration or nutrition (eg, percutaneous endoscopic gastrostomy tubes) $(\mathrm{P}<001)$; and discussing prognosis specifics $(\mathrm{P}<001)$. At 1 -year follow-up, significant improvement remained in 4 of the 5 circumstances compared to baseline: delivering bad news $(\mathrm{P}=0.010)$; discussing hospice or palliative care referral $(\mathrm{P}=0.006)$; discussing artificial hydration or nutrition $(\mathrm{P}=0.002)$; and discussing prognosis specifics $(\mathrm{P}=0.009)$.

Conclusion: In this sample, participation in the Teaching Primary Palliative Care Training program increased health care provider comfort in patient communication of various circumstances related to the management of seriously ill and dying patients. Improvements continued 1 year after the training. Next steps include determining whether increased comfort translates into changes in provider behavior such as conducting goals of care conversations with patients. 


\section{Medication Refill Protocol at a Residency Clinic}

Carl M. Bellinger, Patrick Foss, Fabiana Kotovicz, Dennis J. Baumgardner, Marianne Klumph, Jessica J. F. Kram

Family Medicine, Aurora St. Luke's Medical Center; Aurora UW Medical Group; Center for Urban Population Health

Background: In primary care clinics, prescription refill requests significantly impact day-to-day clinic workflow and can increase clinician burnout and patient dissatisfaction. Delays in response cause gaps in treatment leading to potential patient adverse events and stress on patient/provider relationships.

Purpose: To decrease the time to complete refill requests (refill approved or declined) and improve consistency in management of refills.

Methods: We created an interdisciplinary refill protocol based on state Medicaid preferred drug list data to allow for centralized, nursing-driven management of most prescription refills at a family medicine residency clinic in Milwaukee, Wisconsin. Medication refill processing time (from the time a refill encounter was open with a refill request to the time it was closed with refill approval or denial) was measured in minutes and was compared preprotocol (March 1, 2017, to November 30, 2017), during protocol adoption (December 1, 2017, to August 31, 2018), and postprotocol (September 1, 2018, to June 28, 2019). Mood median test was used to compare the average time for a medication refill request to be addressed. Levene's test was used to test for equal variance surrounding the median of each group.

Results: Cohorts included preprotocol $(\mathrm{n}=24,073)$, protocol adoption $(n=23,770)$, and postprotocol $(n=25,770)$. We found a statistically significant reduction in median time to refill completion, from 232 minutes in the preprotocol phase to 157 minutes in the postprotocol phase $(\mathrm{P}<0.001)$. Reduction in median time to response was most apparent in the resident subgroup, with median times of 383 minutes preprotocol and 79 minutes postprotocol $(\mathrm{P}<0.001)$. There also were statistically significant reductions in variability of response time. Response time standard deviations (SD) had an overall reduction across groups (preprotocol mean SD: 14.5 days; during protocol adoption mean SD: 10.8 days; postprotocol mean SD: 6 days; $\mathrm{P}<0.0001$ ).

Conclusion: This project reinforces the importance of a standardized multidisciplinary medication refill protocol to increase consistency and decrease medication refill time. Results from this project are being used to further improve and expand the process of medication refill within both of our academic family medicine clinics.

\section{Substance Use Disorder Treatment Outcomes and Transitions at a Large Midwestern Health Care System}

Mindy R. Waite, Kayla Heslin, Jonathan Cook, Elizabeth Wanninger, Michelle Simpson, Andrew J. Freeman

\section{Advocate Aurora Research Institute; Aurora Behavioral Health Services}

Background: Substance use disorder (SUD) is diagnosed in $10 \%$ of the U.S. population across a lifetime. Although around $10 \%$ of individuals with SUD go into long-term remission each year, the disease is considered chronic and is punctuated by many periods of abstinence, relapse, and eventual long-term remission. Nevertheless, only $35 \%-50 \%$ of individuals with SUD experience long-term remission within 17 years of diagnosis. To account for disease chronicity and varying levels of need over time, several treatment programs exist, each tailored to an individual's current acuity and needs. These treatments include inpatient, residential, partial hospitalization program (PHP), intensive outpatient program, and outpatient treatment. Patients are assessed and referred for a specific level of treatment, and individual outcomes are superior if patients enter and complete the recommended program. However, many patients choose to reduce their lengths of stay, discharge against medical advice, or enter programs mismatched with their current acuity.

Purpose: This retrospective study describes patient populations within the 5 unique SUD programs as well as variables related to program completion and length of stay.

Methods: Medical records were used to collect data. Eligible subjects were adults enrolled in SUD treatment at a midwestern psychiatric hospital between January 1, 2017, and November 20, 2019. Data included demographics, diagnoses, tobacco use, number and length of stays, referrals, discharge against medical advice, and program type. Basic descriptive statistics were conducted.

Results: Of the total population ( $\mathrm{N}=4990)$, the majority of patients receiving treatment for SUD were male (59.9\%), current smokers $(61.8 \%)$, and had a mean age of 39.9 years. Further, many patients had concurrent mental health diagnoses; depression and/or anxiety disorders were prevalent in $53 \%-57 \%$ of patients. Within the context of substance use, patients were most likely to have been diagnosed with alcohol use disorder, opioid use disorder, cocaine use disorder, and cannabis use disorder. Similarly, patients were most likely to receive treatment for alcohol use, cannabis use, opioid use, and cocaine use. Co-occurring diagnoses were extremely common, and patients in PHP programming had the greatest number of diagnoses.

Conclusion: Patients receiving treatment for substance use disorder are highly varied, and population differences exist among programs. These data suggest opportunities for tailoring programs to meet the current and long-term needs of patients.

\section{Concordant Genomic Vulnerabilities of Patient-Derived Cell Line and Matched Xenograft-Derived Cell Line Through Whole Exome Sequencing in Breast-to-Brain Metastatic Cancer}

Jun Yin, Aspen Duffin, Kate Dennert, Deborah L. Donohoe, Parvez Akhtar, Richard A. Rovin, Judy A. Tjoe

Advocate Aurora Research Institute; Aurora Neuroscience Innovation Institute, Aurora St. Luke's Medical Center; Surgical Oncology, Aurora Sinai Medical Center

Background: Brain metastasis (mets) is often associated with the worst prognosis among all disseminated disease, and one of the two main sources of brain mets is the breast, with reported 1-year survival rate of $20 \%$ once developed. Breast-to-brain mets (BBM) usually happens in a late stage of breast cancer, and options of treatment are very limited, which becomes a major limitation of life expectancy. Therefore, developing a cost-efficient, robust model that could precisely recapitulate the features of tumor origin would be beneficial to discovery of novel therapeutic strategies to further improve outcomes.

Purpose: Patient-derived xenografts (PDXs) often accurately recapitulate the tumor of origin in terms of genomic landscape, histopathology, and therapeutic response; however, restrictions 
such as cost, high maintenance, and limited amenability for large-scale screening for potential therapies remain a challenge. To overcome these issues, we established a platform to derive cell lines from both a patient's BBM tumor and the matched PDX to further investigate the similarity of their pathogenic genomic variants.

Methods: DNAs from the first passage of cells derived from patient tumor along with 2 different passages of cells derived from PDXs were extracted, followed by whole exome sequencing analysis. Raw FASTQ files were quality controlled using FASTQC and then aligned to hg38 using BWA-MEM without trimming. The aligned BAM files were processed using GATK4, following best practice. Mutations were called using mutect 2 without normal control. Classification of variants was finally referred to ClinVar.

Results: Cells derived from PDXs (PDXL) has a significantly shorter doubling time than cells derived from patient tumor (PTL). PDXL was able to recapitulate the entire spectrum of PTL's pathogenic variants. Exome sequencing analyses delineated several pathogenic variants of some common oncogenes and tumor suppressors, which potentially contributed to progression of this tumor. PDXL and PTL have shown consistent response to drugs that target the certain pathogenic variants.

Conclusion: Our patient tumor-PDX cell line platform represents a preclinical tool for functional gene validation and proof-of-concept studies to identify novel druggable vulnerabilities in BBM, which could be further applied to other types of brain mets.

\section{Gynecologic Cancer Patients' Interests in Music Therapy}

\section{Stephanie N. Veit, Jessica J. F. Kram, Kayla Heslin, Elizabeth} L. Dickson Michelson

\section{Obstetrics and Gynecology, Aurora Sinai Medical Center; Aurora UW Medical Group; Center for Urban Population Health; Advocate Aurora Research Institute; Gynecologic Oncology, Aurora West Allis Medical Center}

Background: Music therapy has been used as complementary therapy in oncology patients undergoing chemotherapy for decades. Several studies have suggested potential benefits associated with music therapy use, including alleviation of mood symptoms like anxiety and other common chemotherapy side effects like nausea and vomiting. Although case reports have reported music therapy use among women with gynecologic cancers, no formal studies have been conducted.

Purpose: To evaluate patients' thoughts on music therapy as an adjunct to chemotherapy and to evaluate patients' use of music therapy in comparison to other media types.

Methods: We conducted a feasibility study that prospectively surveyed adult women undergoing chemotherapy for a gynecologic cancer (endometrial cancer, ovarian cancer, cervical cancer, etc) within 1 hospital center from June 2018 to July 2019. Women were asked demographic and preliminary questions prior to being provided preselected music on an audio listening device. Following their chemotherapy session, women were asked to complete a survey remarking on their thoughts about music therapy and use of other media. Basic descriptive statistics were conducted.

Results: Overall, 20 women completed surveys, of which $50 \%$ were 30-59 years of age, with the remainder greater than 60 years of age. Women were predominately Caucasian (75\%) and mostly had either ovarian $(50 \%)$ or endometrial $(45 \%)$ cancer. Overall, $80 \%$ of women chose to listen to the music provided instead of their own music. Most women (35\%) listened to music for 30 39 minutes during their chemotherapy session. Choice of music genre varied greatly between women. Ultimately, $70 \%$ of women participated in another activity in addition to listening to music, with $85 \%$ choosing to switch to another activity entirely at some point during their chemotherapy session. The most common activities patients engaged in besides music therapy included talking to family and friends $(65 \%)$, social media $(30 \%)$, reading (25\%), and sleeping (25\%). Women found music therapy to be very helpful, relaxing, and a good time-filler.

Conclusion: Although many women listened to music during their chemotherapy, most also engaged in another activity, sometimes switching completely to that other activity. Based on our findings and the difficulties of even surveying patients, participation in a larger study aimed at evaluating effects of music therapy on chemotherapy in women with gynecologic cancers may be quite difficult.

\section{Humans of Family Medicine}

Dane Olsen, Anna E. Karst, Glenda Sundberg, Sarah A. Bowlby, Jessica J. F. Kram

Family Medicine, Aurora St. Luke's Medical Center; Aurora St. Luke's Family Practice Center; Aurora UW Medical Group; Center for Urban Population Health

Background: Primary care providers in Milwaukee are surrounded by a diverse community, often hearing stories of overcoming adversity. Celebrating patients' and staffs' successes are part of what makes medicine so rewarding. The concept of Humans of Family Medicine stems from the Instagram account Humans of New York, which highlights the stories of everyday people in New York City.

Purpose: To highlight and share unique patient and staff stories in hopes of bringing a humanitarian side to the practice of family medicine.

Methods: We conducted a quality improvement project at 2 family medicine clinics within Milwaukee County during 2019. The project was approved by the local institutional review board. Consent was obtained from patients and staff who agreed to have their story told. The stories were written and then vetted by our social media team, before being published on institutional social media accounts. Family medicine providers and staff were surveyed before and after the project to gauge use of social media and thoughts on the project. Pre/postsurvey responses were compared with Fisher's exact tests. P-value less than 0.05 was considered significant.

Results: A total of 25 individuals were asked if they would like to participate and share their story; 1 declined. In all, 24 stories were written; 18 have been published ( 3 on Facebook [FB] alone, 0 on Instagram [IG] alone, and 15 on both platforms). Of the 24 stories, 12 were current patients, 10 were staff members, and 2 were resident physicians. Mean FB likes were 485 (range: 75-1155), and mean IG likes were 96 (range: 45-490). Mean FB comments were 33 (range: 3-173), and mean IG comments were 5 (range: $0-56)$. Family medicine providers and staff $(n=23$ presurvey; $n=28$ postsurvey) were significantly more likely to follow our institution on Facebook following project implementation $(\mathrm{P}=0.005)$. Additionally, family medicine providers and staff were significantly more likely to think it was a good idea to showcase patient and staff stories on social media following the project $(\mathrm{P}<0.001)$. Those surveyed initially were unsure of whether their patients would 
like their stories showcased (36\% "yes, would like"). Following the project, $82 \%$ thought patients would like being showcased on social media $(\mathrm{P}=0.001)$.

Conclusion: Humans of Family Medicine creates a platform to share stories about the people we serve and is something our staff and providers think is a good idea. It is possible that showcasing these stories may bring a more humanitarian side to the practice of family medicine.

\section{Hot Spotting Medically Complex, Socially At-Risk Patients Accomplishes the Quadruple Aim}

Keyonna Taylor-Coleman, Glenda Sundberg, Jessica J. F. Kram

Family Medicine, Aurora St. Luke's Medical Center; Aurora St. Luke's Family Practice Center; Aurora UW Medical Group; Center for Urban Population Health

Background: The goal of the quadruple aim for health care is to 1) improve patients' experiences, 2) address social determinants of health, 3) reduce health care costs, and 4) prioritize the well-being of care team members. Hot spotting, or collaborating with other health care professionals to create a care plan to address our most complex patients, may accomplish the quadruple aim.

Purpose: To illustrate that hot spotting addresses the ideology behind the quadruple aim.

Methods: Across 3 clinic sites in 2019, providers recommended patients who would benefit from the hot spotting intervention (preintervention period: June 1, 2018-February 28, 2019; postintervention period: March 1, 2019-December 1, 2019). Three times per year, interdisciplinary teams at each site completed care plans for each patient. Patients received at least 1 home visit and increased calls from nursing/social work. Social determinants of health were assessed. Hospitalizations and emergency department visits were compared. Pre- and postintervention surveys were conducted by staff and providers involved in hot spotting. Basic descriptive statistics, and Mann-Whitney $U$ test when appropriate, were conducted.

Results: In all, 64 patients were hot spotted. Patients were predominately female (69\%), African American (60\%), Englishspeaking (89\%), and had Medicare/Medicaid insurance $(100 \%)$. Patient ages ranged from 2 to 98 years. Patients lived on average within $3.7 \pm 2.8$ miles to their respective clinics. Addressing the first aim, hot spotting improved the patient experience for $78 \%$ of patients who received additional coordination of care as a result of their involvement that differed from the traditional health care model. Evaluation of the social determinants of health revealed concerns with transportation (39\%), food insecurity (31\%), safe relationships (34\%), financial safety (45\%), and safe housing (36\%), allowing providers to better address these needs during home visits and accomplish the second aim. Moreover, for a third year, although not statistically significant, emergency department visits decreased among hot spots (13.1\% decrease), implying cost savings. Overall, prior to and following the hot spotting intervention, a majority of survey respondents felt that they were able to provide better care to their patients because of the intervention.

Conclusion: Hot spotting accomplishes the quadruple aim by 1) demonstrating improved patient experience, 2) addressing underlying social determinants of health, and 3) reducing health care costs, while 4) showing an overwhelmingly positive care team response to the intervention.
Correlation of Occurrence of Legionella pneumophila and Blastomycosis Cases Within Zip Codes: Eastern Wisconsin

\author{
Dennis J. Baumgardner \\ Department of Family Medicine, Aurora UW Medical Group; \\ Center for Urban Population Health
}

Background: Legionella pneumophila (intracellular bacteria) pneumonia (LpP) and blastomycosis (from the dimorphic fungi Blastomyces) are potentially serious environmentally acquired infections that are both prevalent in Wisconsin. There is good evidence that blastomycosis is associated with waterways and preliminary evidence that some LpP may be as well. If the acquisition of both diseases is similarly associated with a geographic feature, one might expect similar geographic distributions of case addresses. Purpose: To perform a preliminary exploratory analysis of the correlation of the distribution of LpP and blastomycosis cases among zip codes in eastern Wisconsin.

Methods: We conducted secondary data analyses of 2 ACL Laboratories registries covering eastern Wisconsin from overlapping 5-year time periods: positive L. pneumophila urine antigen tests (LUAT), which fit Centers for Disease Control and Prevention criteria for diagnosis, from 2013 to 2017, and laboratory-confirmed blastomycosis cases from 2015 to 2019. Number of respective cases, by zip code, were compared for zip codes $530 \mathrm{xx}-532 \mathrm{xx}$ and $534 \mathrm{xx}$ containing 5 or more LUAT results. One zip code outlier was eliminated. Incidence figures for each disease were calculated from our previously published analysis data. Pearson correlation was calculated, and linear regression was performed with LpP case distribution as outcome variable and blastomycosis distribution as predictor.

Results: Yearly predicted LpP and blastomycosis cases in the eastern Wisconsin catchment area were 27 and 24, respectively, such that an approximately 1:1 distribution was assumed. Pearson correlation of distribution of LpP and blastomycosis cases by zip code was moderate at $0.541(\mathrm{P}<0.001)$. Of 136 zip codes from 11 counties studied, 61 had no LpP or blastomycosis cases, 35 both types of cases, 24 only LpP cases, and 16 only blastomycosis cases. Blastomycosis case distribution was a significant predictor of $\mathrm{LpP}$ cases in a linear regression model $(\mathrm{P}<0.001)$, with equation LpP cases by zip $=0.419$ +0.636 (number of blastomycosis cases); adjusted $\mathrm{R}^{2}=28.7 \%$.

Conclusion: This preliminary, modest correlation of the zip code distribution of L. pneumophila pneumonia and blastomycosis is intriguing given known association of LpP with human-built water sources. It may suggest an undescribed common outdoor environmental source. Further study of LpP and blastomycosis coassociations with waterways, and other potential common sources (or common environmental hosts such as Acanthamoeba in which both can propagate), seems warranted.

\section{Identifying the Value of Graduate Medical Education to a Health Care System}

Deborah Simpson, Thomas J. Hansen, Jacob Bidwell, Mary Joyce Turner

Family Medicine, Advocate Aurora Health; Academic Affairs, Advocate Aurora Health; Aurora Graduate Medical Education, Advocate Aurora Health 
Background: Our newly merged health care system sponsors more than 650 residents and fellows in our accredited programs. Sponsoring graduate medical education (GME) programs requires a significant financial and human investment from multiple parts of the organization - ranging from executive leadership/board/Csuite/hospital leaders to leaders responsible for human resources, legal, finance, quality, and safety.

Purpose: To identify what system leaders perceived to be GME's value to our health care system.

Methods: Our GME leadership team developed brief questions seeking to have interviews of $<20$ minutes: 1 ) When you need to advocate for the value of our GME programs, what do you highlight? and 2) What do you wish others would value about our GME programs? Three authors were assigned to contact a key system leader through email, phone call, or face-to-face. Project purpose was explained (eg, interested in their perceptions in regard to the value of GME to our organization) and leader asked if they would be willing to meet with us. A field notes worksheet was created for interviewers to note key findings. Respondent's leadership role (eg, executive team, C-suite, hospital president/chief medical office, finance/legal) was recorded, along with their responses to each question. Responses were then coded and categorized using standard qualitative methodology to identify cross-cutting themes.

Results: Of 31 leaders approached, 29 (94\%) agreed to be interviewed, with field notes analyzed. The top 4 areas our system leaders highlighted when advocating for GME, with little/no disagreement by leadership role, were: 1) its value and cost-effectiveness as a pipeline for physician recruitment; 2) GME's contribution to a culture of continuous learning; 3 ) the prestige/reputation associated with being an organization that trains future physicians; and 4) the expectations of the community and the profession to train the next cohort of physicians. Typical response was that residents create a "healthy tension in the organization. Organizations are built to just 'do,' not to create questions/reflect ... it wouldn't happen without education programs." All 4 of these areas matched what leaders wished others valued about our GME programs, with 1 area added: the infrastructure needed to support education.

Conclusion: Identifying graduate medical education's value beyond a workforce pipeline allows GME leaders to emphasize GME's broader assets to the organization. Our next step is to engage program directors on metrics for these values and share those findings to further enhance GME's value to the organization.

\section{Radiation Exposure, Reduction Techniques, and Standardization of Swallow Study Evaluations}

Mason A. Brown, Shelly Reimer, Leah Presper, Theresa Acherman, William MacDonald

Diagnostic Radiology, Aurora St. Luke's Medical Center; Speech Pathology, Advocate Aurora Health; Radiology, Advocate Aurora Health

Background: Long-term exposure to ionizing radiation from fluoroscopic procedures can lead to deterministic and stochastic effects not only for the patient but for medical personnel as well. Swallow study evaluations are the most frequent fluoroscopic procedures performed and often require a speech pathologist, fluoroscopy technician, and radiology resident to be present in the room during the study. While $\mathrm{C}$-arm radiography machines calculate the estimated absorbed radiation dose to the patient, cumulative radiation exposure badge data and baselines for medical personnel are difficult to interpret, track, and maintain.

Purpose: Establishing a fluoroscopic radiation exposure baseline in radiology residents may help in estimating other medical personnel exposure and monitoring future reduction techniques.

Methods: For the baseline radiation exposure analysis, fluoroscopic study data were retrospectively collected. Powerscribe is a radiology dictation software in which study reports are organized, and we sorted each resident's studies into a database. Swallow study absorbed dose, which is ionizing radiation absorbed per unit mass measured in grays (Gy), is recorded with respect to the patient's dosing. This amount was used to estimate the radiology resident's radiation exposure utilizing an intensity calculated based off a distance of 2.5 $\mathrm{m}$. Radiation exposure data were analyzed per individual residents in the current PGY3 class. Future methods include implementing reduction techniques and analyzing radiation exposure data in the current PGY2 class.

Results: Patient radiation exposure data per swallow study evaluation averaged 1.9 minutes (median: 1.8 minutes, range: $0.3-$ 4.3), where the average radiation exposure was 7.9 mGy (median: $7.2 \mathrm{mGy}$, range: $1.5-24.3)$. Residents performed approximately 5 swallow studies/day, or 100 swallow studies/4-week rotation. By utilizing an intensity calculation of Intensity $=1 /$ distance $^{2}$, estimated resident radiation exposure over 8 weeks $(2 \times 4$-week rotations) was 367.7 minutes (6.1 hours) and $23.9 \mathrm{mGy}$.

Conclusion: In residents, exposed areas of skin receive an ionizing radiation dose equivalent to 3 computed tomography scans per 4-week fluoroscopy rotation. Eye lenses have a deterministic threshold of 2-6 Gy. Residents absorb more than $10 \%$ of the lower end of this threshold during their first year of radiology training. Speech pathologists and fluoroscopy technicians without protective equipment could be at similar risk levels.

\section{Improving Timeliness of Early Hospital Discharges: A Quality Improvement Project}

Itamar Gnatt, Alice Shallcross, Leart Neziri, Jessica J. F. Kram, Javier Leiva, Marianne Klumph, Fabiana Kotovicz

\section{Family Medicine, Aurora St. Luke's Medical Center; Aurora UW Medical Group; Center for Urban Population Health; Performance Advisory, Advocate Aurora Health}

Background: Delayed hospital discharges create bottlenecks in health care systems, increase health care costs, backlog emergency departments, and leave hospitalized patients susceptible to infections and emotional distress. Several studies have identified barriers to and interventions for discharge timeliness in order to decrease the impact of delayed patient discharges.

Purpose: This quality improvement project aimed to explore and identify barriers to early discharge for patients within one hospital teaching service.

Methods: From August 2019 to November 2019, semi-structured interviews with family medicine faculty, residents, and hospital nurses were performed and recorded to further explore barriers and opportunities to early (before noon) discharge within a tertiary academic hospital in urban Milwaukee, Wisconsin. Transcribed interviews were further reviewed using qualitative software Dedoose 8.3.17 for thematic inductive analysis. Additionally, an online survey for residents was distributed and basic descriptive statistics calculated to summarize survey responses.

Results: Through qualitative analyses, 4 themes were identified. Overarching themes included: 1) barriers to early discharges (eg, 
suboptimal communication [components cited 87 times during interviews], safety concerns [cited 4 times], language/transportation barriers [cited 60 times], specialty clearance [cited 20 times]); 2) suggestions for improvement (eg, electronic health record tools, examples of good communication, clear expectations, waiting lounge, process standardization); 3) discharge planning process (eg, timely medicine reconciliation and order [cited 17 times], patient discharge expectations [cited 15 times]); and 4) system implications of discharge by noon (eg, workflow improvement, communication improvement).

Conclusion: Our findings highlight the need for standardization of discharge workflow and potentially modifiable early-discharge barriers. Results from this study will be used to implement interventions and educate providers on ways to optimize early hospital discharges.

\section{Group Visits in Chronic Disease Management}

Dane Olsen, Christina Quale, Fabiana Kotovicz, Jessica J. F. Kram, Marianne Klumph, Karen Pankowski

Family Medicine, Aurora St. Luke's Medical Center; Aurora St. Luke's Family Practice Center; Aurora UW Medical Group, Center for Urban Population Health

Background: Chronic disease management encompasses most of a primary care provider's practice. However, brief office visits often pose a challenge to provide comprehensive patient education for their chronic conditions. One modality thought to improve management of chronic diseases is the group medical visit model. Even so, despite proven benefits for patients and medical providers, creating a sustainable chronic disease group is challenging.

Purpose: To identify the benefits and challenges of developing and maintaining a group visit model at academic primary care clinics in urban underserved settings.

Methods: Semi-structured interviews $(\mathrm{N}=9)$ with team members who had previously led or participated in group sessions for chronic disease management at 2 academic primary care clinics in Milwaukee, Wisconsin, were performed and recorded from October 2018 through March 2019. Interviews were reviewed by 2 different investigators who independently coded transcriptions using inductive methods using qualitative software (Dedoose v.8.3.17). Codes generated independently were then integrated into a single codebook by a third investigator.

Results: Three major themes were identified from the interviews: 1) Expected and actual benefits (eg, overall patient and provider satisfaction [ $56 \%$ of interviewees identified], patient empowerment [67\%], more time with patients [34\%]); 2) Expected and actual challenges (eg, recruitment [78\%], retention [100\%], scheduling of providers [45\%], language barriers [23\%], poor health literacy [34\%]); and 3) Advice for providers conducting future group visits (eg, establishing a consistent leader to champion the group visit [23\%], develop a well-organized curriculum [56\%], understand reimbursement [23\%]).

Conclusion: Group medical visits have several inherent barriers, especially around recruitment and retention. Even so, interviewees identified that group medical visits are beneficial to both patients and providers. Given the identified potential patient benefits, such as patient empowerment and improved health literacy, findings from our qualitative study will be used to identify resources to promote group visit success.
Blood Lead Screening Rates in Children Aged 12-35 Months Within a Milwaukee Family Medicine Residency Clinic

Priti Rana, Morgan Lively, Marianne Klumph, Do Dang, Bonnie Bobot, Alonzo Jalan

Family Medicine, Aurora St. Luke's Medical Center; Aurora UW Medical Group; Center for Urban Population Health; University of Wisconsin School of Medicine and Public Health; Family Medicine, Aurora Sinai Medical Center

Background: There is no safe level of lead in the body. Elevated blood lead levels primarily affect the development of the central nervous system. Blood lead levels are highest between the ages of 18 and 36 months secondary to an increase in mobility and hand-tomouth behavior. Children in the city of Milwaukee are recommended to be screened for lead levels at 12, 18, and 24 months, followed by annual screening through the age of 5. Yet, Milwaukee continues to have a greater proportion of children who test positive for elevated lead levels compared to the national average.

Purpose: To review the rate of lead screening in children aged 12-35 months at Aurora Sinai Medical Center's Family Care Center (FCC) clinic and to identify risk factors.

Methods: Data were retrospectively collected from Aurora Health Care's electronic health records and the Wisconsin Lead Registry on children 12 to 35 months old who attended FCC for well-child exams from October 1, 2018, to September 30, 2019. The screening results were sorted into the age groups of 12-17, 18-23, and 24 - 35 months, giving us a snapshot of screening rates. Basic descriptive statistics were computed, and Fisher's exact test was used for categorical analysis.

Results: A total of 383 patients were included for analysis. In all, there was an equal percentage of females and males; $72 \%$ were Black, followed by $10 \%$ White, and 10\% Hispanic. Appropriate lead level screening rates of children by age were as follows: $45 \%$ for 12-17 months, $26 \%$ for $18-23$ months, and 35\% for 24-35 months. Overall, $62 \%$ of $12-35$-month-old children at FCC had at least one blood lead test. There was no statistically significant difference in screening based on race as defined by Black vs non-Black $(\mathrm{P}=0.56)$. However, there was a statistically significant difference between insurance carriers, with Medicaid patients being screened more than those with other types of insurance $(\mathrm{P}=0.016)$.

Conclusion: Lead screening rates at FCC were comparable to statewide screening. The higher screening rate for Medicaid patients may likely be due to having additional access to WIC services and recommended point-of-care lead testing. Implementation of point-ofcare lead testing at FCC may improve overall future screening rates.

\section{Adoption of Macy Catheter by Aurora at Home Hospice Staff: A Quality Improvement Evaluation Study}

Patrick B. Herson, Kavita Sharma, Kristi Barfield, Nora Clapsaddle

Hospice and Palliative Care Medicine, Aurora St. Luke's Medical Center; Hospice Care, Aurora Health at Home

Background: The Macy Catheter (MC) is a tool to aid rectal delivery of oral medications in microenemas. In 2019, Aurora Health at Home hospice chose the MC as a preferred route for delivery of medications when patients no longer tolerate the oral route. Despite training, adoption of the MC has been slow. 
Purpose: To determine what barriers exist to faster adoption and utilization of the MC by hospice nursing staff. It is anticipated that once the barriers are identified they will be addressed through a campaign of quality improvement projects with subsequent monitoring of adoption.

Methods: An anonymous survey was given to 28 nurses at 2 meetings. The survey identified nurses that used the MC and their experiences. Nurses who had not used it were queried to see if they had no eligible patients or, if they had eligible patients, what their reasons were for not using it. Demographic data included age, experience as a registered nurse ( $\mathrm{RN})$ and hospice $\mathrm{RN}$, usual shift worked, and work status. Basic descriptive statistics were used to describe the population.

Results: Of a total of 28 surveys completed, 7 reflected use of the MC, with most using it only once and 1 using it 3-5 times. The number of medication doses given ranged from 1 to 10-20. There was an overall agreement regarding the ease of the $\mathrm{MC}$ insertion and its effectiveness when compared to the oral route. There were no complications noted. Ten nurses reported to never have used the MC because of not having eligible patients, while 12 reported having eligible patients and not using the $\mathrm{MC}$, though 9 considered it. Common reasons for not using it include patient/family objections, inexperience using the MC, or confidence in other methods. One nurse reported not using it due to a nursing facility policy. Of those who had eligible patients and who did not consider using it, reasons given included unfamiliarity and not thinking of it in the "heat of the moment." There were no demographic differences among these groups that would account for their willingness to use or consider the MC.

Conclusion: The study identified leadership opportunities to increase the use of the Macy Catheter. Experienced nurses can help train and teach their peers how to "sell" this device to patients, families, and other clinicians. There is a need to develop educational collateral to help families understand the utility of the MC. We can work with facilities to make sure their policies align with the latest nursing approaches for excellent hospice care.

\section{Medical Students Add Value in Your Clinical Setting}

\author{
Deborah Simpson, Mark Robinson, Kjersti Knox, Theresa \\ Frederick, John R. Brill
}

Family Medicine, Advocate Aurora Health; Clinician Student Services, Academic Affairs, Advocate Aurora Health; Training in Urban Medicine and Public Health (TRIUMPH), University of Wisconsin School of Medicine and Public Health

Background: First- and second-year medical students perceive that they can make meaningful contributions to patient care and/or the health care team's learning. However, there is limited formal data from more senior medical students regarding the value they add to patient care and/or the team during their late-second through fourth year-required clinical rotations.

Purpose: To obtain and codify senior medical students' perceptions regarding the value they add to patient care and/or the health care team during their required clinical rotations.

Methods: Senior medical students at end of required clinical rotations voluntarily completed an online, anonymous 5-item "value added to clinical care" survey. Medical students checked if they added value to 1 of 5 literature-derived categories and then were asked to describe the situation in HIPAA-compliant format. Analysis included frequency tabulations by value-added category and qualitative analysis of narrative examples by category.
Results: There were 131 respondents (53\% women), $80 \%$ of whom were within 12-24 months of graduation. In all, $67 \%$ of valuedadded contributions occurred in the outpatient setting, 23\% in the inpatient setting, and $10 \%$ in other settings (eg, 50/50 inpatient/ outpatient and emergency department). More than half of reports (53\%) focused on the category Patient's/Family's Experience of Their Care (eg, enhancing patient's understanding of their clinical situation). For example, "I had a visit (with patient) who had a gynecological concern but also had recently lost her twin sister. I addressed her chief complaint but then spent some extra time talking to her about how she is handling her loss and the grief she is experiencing." In other categories, $24 \%$ focused on added value to Clinical Quality (eg, obtaining data/information from the patient that impacted a clinical decision, identifying alternative approaches), $18 \%$ on Preceptor/Team Learning, and 3\% of students reported Patient Safety-related valued-added situations. (eg, shared a patient safety concern/a potential risk).

Conclusion: Identifying the value added by students allows educators to identify assets and potential opportunities (like patient safety) to enhance student roles in clinical care. It also provides rich data for educational leaders' use with key clinical/community stakeholders regarding how medical student education adds value to patient care and the health care team.

\section{Younger Hypertensive Patients Are More Likely to Have Activated MyChart Accounts}

Erin Harvey, Will Lehmann, Chella Bhagyam, Keyonna TaylorColeman, Catherine de Grandville, Deborah Simpson

Family Medicine, Aurora St. Luke's Medical Center; Academic Affairs, Advocate Aurora Health

Background: National epidemiological studies note that young adults, those under 39 years old, tend to have less awareness of their hypertension (HTN) diagnosis and are less likely than older adults to have their blood pressure under control. Proposed barriers to hypertension control in young adults include provider reluctance to diagnose hypertension, hesitancy to prescribe antihypertensives, less access to primary medical care, and patient perceptions of their health status. HTN, as a silent disease, has major long-term consequences on cardiovascular health. Identifying HTN patients by age and seeking to control it is an important role for primary care.

Purpose: To determine if the percentage of patients with diagnosed uncontrolled HTN who have signed up for MyAurora differs by age (18-49 years, $\geq 50$ years) and if patients on MyAurora were more likely to have controlled their HTN.

Methods: Data were abstracted from electronic health records, using the BI Launch Pad (the data warehouse for Aurora Quality Improvement), for HTN by age, blood pressure control status, and MyAurora account activation. Data were reviewed by each of two family medicine residency clinic and then aggregated across the two clinics using descriptive statistics.

Results: At one residency clinic, 29\% (46 of 156) of 18-49-yearold adults had uncontrolled HTN; at the second clinic, 31\% (43 of 139) of the same-aged patients had uncontrolled HTN. Rate of adults $\geq 50$ years old who had uncontrolled HTN was $18 \%$ (144 of 816 ) and $23 \%$ ( 85 of 364 ), by respective clinic. When comparing account activation for MyAurora across our two clinics, we found $49 \%$ of patients $18-49$ years old with diagnosed HTN had signed up while only $27 \%$ of patients $\geq 50$ years old with diagnosed HTN had activated their accounts. Across our two clinics, across all ages, 
hypertensive patients who had signed up for MyAurora were 2.3\% more likely to have blood pressure control; however, in the younger adult hypertensive population, patients signed up for MyAurora were $8.1 \%$ more likely to have controlled blood pressure compared to their unenrolled peers.

Conclusion: Consistent with the literature, the percentage of patients with uncontrolled hypertension decreases with age in our primary care clinics. Younger hypertensive patients (18-49 years of age) were more likely to sign up for MyAurora. Higher MyAurora utilization rates for this age group points to its potential use as an intervention for improving blood pressure control in these hypertensive adults.

\section{Quality Improvement Project to Increase Breast Cancer Screening in an Internal Medicine Clinic}

Michael Peliska, Dereje Siyum, Zeba Shethwala, Moaz Hamed, Matt Boese, Hassan Homida, Hina Mahboob, Biana Leybishkis

Internal Medicine, Aurora Sinai Medical Center; Aurora UW Medical Group

Background: Breast cancer is the most common non-skin cancer among American women, accounting for $7 \%$ of all cancer deaths each year. Fortunately, when detected early with regular screening mammograms, the 5-year survival rate for breast cancer approaches $99 \%$. Resident clinics such as ours that serve an underserved urban population bear the brunt of this challenge.

Purpose: To improve the completion percentage of screening mammograms in our underserved urban community from $73 \%$ to $80 \%$. We looked to see if primary care providers and clinic staff could utilize the methods as described herein as effective ways to improve breast cancer screening rates in our community.

Methods: We sought to achieve this goal by reviewing our patient's records to find individuals with a care gap specifically regarding annual breast cancer screening. Once identified, we would personally telephone eligible patients once per week to discuss their breast cancer screening status, explain the risks and benefits of screening mammograms, and encourage them to perform appropriate testing. If they were agreeable, patients were provided a phone number to call and schedule their mammogram. Additional phone calls and mail reminders were sent to patients who agreed to complete mammograms as a way to follow up and encourage timely completion of the screening test.

Results: The initial 6-month data indicated a change in our breast cancer screening completion percentage from $73 \%$ to $77 \%$. Just 1 phone call per provider per week led to a $4 \%$ improvement in our breast cancer screening completion rate. This outcome is preliminary, and we are aiming for more significant improvement in the following months.

Conclusion: Our findings indicate that identifying and contacting patients who are overdue on their preventive health measures, specifically screening mammograms, can have a measurable impact. While the COVID-19 pandemic did affect our abilities to reach our original goal, the results thus far are promising and suggest that with continued effort our $80 \%$ goal is reachable.

\section{Measures to Enhance Breast Cancer Screening}

\author{
Payal Sharma, Ifreah Usmaiel, Jalel Hunde, David Hamel
}

Internal Medicine, Aurora Sinai Medical Center; Aurora UW Medical Group

Background: Breast cancer is responsible for the deaths of more than 40,000 women in the United States. The U.S. Preventive Services Task Force (USPSTF) recommends biannual mammograms for all women 50-74 years of age. Today, the 5-year survival of breast cancer is almost $90 \%$, compared to $75 \%$ in 1975 . Breast cancer screening (BCS) leads to earlier detection and treatment, reduced mortality, and reduced need for toxic treatments. Despite the documented screening benefits, only $64 \%-81 \%$ of the population meeting the criteria for regular BCS is regularly screened.

Purpose: The USPSTF guideline for BCS is a quality improvement (QI) measure that is tracked for primary care physicians (PCPs) in Advocate Aurora Health. PCPs in our clinic had a BCS rate of $67 \%$ for the eligible patients assigned to them in September 2019 (system goal: $\geq 84 \%$ ). We hypothesized that regular BCS has been limited by factors including inadequate emphasis, education, and reminders. By April 1, 2020, our PCPs aim to fill these gaps and improve patient outcomes by increasing our BCS rate by $\geq 10 \%$.

Methods: The study population included our clinic PCPs (authors P.S., I.U., J.H.) who performed personal outreach to their patients due for BCS. Every month, data were generated through the system's QI process, which included percentage of eligible BCS patients who were up-to-date and a list of patients who were due. The process for the study was our routine clinic workflow, plus dedicated outreach to our patients (ie, the intervention). We called, mailed letters, or messaged through our electronic messaging system (MyAdvocateAurora) to our patients who were due for BCS. We advised our patients to complete a screening mammogram given supporting facts. A screening mammogram was ordered for those who agreed. We provided the patients with a phone number to schedule it. For patients who had BCS in other systems, we collected the results through the usual process and updated it in our system.

Results: At the start of our QI projects, only 67\% of 50-75-year-old patients had up-to-date BCS. By continuous and dedicated outreach, our BCS score has improved to $75 \%$ by February 20, 2020.

Conclusion: To improve breast cancer screening rates and decrease breast cancer-related mortality, we established individualized outreach. These efforts increased QI measures. However, our project was not able to continue until April 1, 2020, as planned due to indefinite closure of nonessential clinic visits starting at the end of February 2020, due to the COVID-19 pandemic. Even though we were not able to continue the project, by continuing these dedicated outreach reminders, we might have been able to reach our BCS rate goal of $\geq 77 \%$ if it were not for the pandemic.

(C) 2020 Advocate Aurora Health, Inc. 